PROCEEDINGS OF THE

AMERICAN MATHEMATICAL SOCIETY

Volume 139, Number 10, October 2011, Pages 3735-3738

S 0002-9939(2011)11015-3

Article electronically published on May 24, 2011

\title{
ON EXPLICIT PROBABILITY DENSITIES ASSOCIATED WITH FUSS-CATALAN NUMBERS
}

\author{
DANG-ZHENG LIU, CHUNWEI SONG, AND ZHENG-DONG WANG
}

(Communicated by Jim Haglund)

\begin{abstract}
In this paper we give explicitly a family of probability densities, the moments of which are Fuss-Catalan numbers. The densities appear naturally in random matrices, free probability and other contexts.
\end{abstract}

In this paper we study a family of probability densities $\pi_{s}, s \in \mathbb{N}$, which are uniquely determined by the moment sequences $\left\{m_{0}, m_{1}, \ldots, m_{k}, \ldots\right\}$ [1]. Here

$$
m_{k}=\frac{1}{s k+1}\left(\begin{array}{c}
s k+k \\
k
\end{array}\right)
$$

are known as Fuss-Catalan numbers in free probability theory [7. The densities $\pi_{s}$ belong to the class of free Bessel laws 3 and are known to appear in several different contexts, for instance, random matrices [1, 3, 7, random quantum states [4, free probability and quantum groups [3, 6. More precisely, $\pi_{s}$ is the limit spectral distribution of random matrices in the forms such as $X_{1}^{s} X_{1}^{* s}$ and $X_{1} \cdots X_{s} X_{s}^{*} \cdots X_{1}^{*}$, where $X_{1}, \ldots, X_{s}$ are independent $N \times N$ random matrices (the matrix elements of different matrices are independent); in free probability we have the free convolution relation: $\pi_{s}=\pi_{1}^{\bigotimes s}$ [7, 3].

More generally, T. Banica et al. 3 introduce a remarkable family of probability distributions $\pi_{s, t}$ with $(s, t) \in(0, \infty) \times(0, \infty)-(0,1) \times(1, \infty)$, called free Bessel laws. $\pi_{s}$ is the special case where $t=1$, i.e., $\pi_{s, 1}=\pi_{s}$. The moments of $\pi_{s, t}$ are called the Fuss-Catalan polynomials (Theorem 5.2, 3]):

$$
m_{k}(t)=\sum_{j=1}^{k} \frac{1}{j}\left(\begin{array}{l}
k-1 \\
j-1
\end{array}\right)\left(\begin{array}{c}
s k \\
j-1
\end{array}\right) t^{j} .
$$

Indeed, the following relation holds $[3]$ :

$$
\pi_{s, 1}=\pi_{1}^{\bigotimes s}, \quad \pi_{1, t}=\pi_{1}^{\boxplus t} .
$$

The distribution $\pi_{1, t}$, the famous Marchenko-Pastur law of parameter $t$, also called free Poisson law, has an explicit formula:

$$
\pi_{1, t}=\max (1-t, 0) \delta_{0}+\frac{\sqrt{4 t-(x-1-t)^{2}}}{2 \pi x} .
$$

Received by the editors August 4, 2010.

2010 Mathematics Subject Classification. Primary 60B20, 46L54; Secondary 05A99.

Key words and phrases. Fuss-Catalan numbers, moments, free Bessel laws.

(C)2011 American Mathematical Society 
In the special case,

$$
\pi_{1}=\pi_{1,1}=\frac{1}{2 \pi} \sqrt{4 x^{-1}-1}
$$

Another special case of $\pi_{s, t}$ where an explicit density formula is available is due to Penson and Solomon $[8]$

$$
\pi_{2}=\pi_{2,1}=\frac{\sqrt[3]{2} \sqrt{3}}{12 \pi} \frac{\sqrt[3]{2}(27+3 \sqrt{81-12 x})^{2 / 3}-6 \sqrt[3]{x}}{x^{2 / 3}(27+3 \sqrt{81-12 x})^{1 / 3}} 1_{(0,27 / 4]}(x) .
$$

To the best of our knowledge, except for the special cases above there are no explicit formulae available for the other $\pi_{s, t}$. The aim of this work is to give explicit densities of $\pi_{s}=\pi_{s, 1}, s \in \mathbb{N}$. The proof of the following result is based on the method to find an explicit density from a given moment sequence used in 9 , 5].

Theorem 0.1. Let $\pi_{s}, s \in \mathbb{N}$, be the unique densities determined by the FussCatalan numbers in Eq. (0.1). Then we have the following formulae:

$$
\pi_{s}(x)=\frac{1_{(0, K]}(x)}{B\left(\frac{1}{2}, \frac{1}{2}+\frac{1}{s}\right)} \int_{[0,1]^{s}} \frac{(\tau K-x)^{1 / s-1 / 2}}{\sqrt{x}(\tau K)^{1 / s}} F\left(t_{1}, \ldots, t_{s}\right) 1_{\{\tau K \geq x\}} d^{s} t,
$$

where $K=(s+1)^{s+1} / s^{s}, \tau=\prod_{j=1}^{s} t_{j}$ and $F\left(t_{1}\right)=\delta\left(t_{1}-1\right)$, while for $s>1$

$$
\begin{aligned}
F\left(t_{1}, \ldots, t_{s}\right) & =\frac{1}{B\left(\frac{1}{s+1}, \frac{s-1}{2 s+2}\right) \prod_{j=2}^{s} B\left(\frac{j}{s+1}, \frac{j}{s(s+1)}\right)} \\
& \times t_{1}^{\frac{1}{s+1}-1}\left(1-t_{1}\right)^{\frac{s-1}{2 s+2}-1} \prod_{j=2}^{s} t_{j}^{\frac{j}{s+1}-1}\left(1-t_{j}\right)^{\frac{j}{s(s+1)}-1} .
\end{aligned}
$$

Proof. First, we derive a family of symmetric distributions $\sigma_{s}$, the $2 k$-moments of which are $m_{k}$ in Eq. (0.1).

Consider the characteristic function of $\sigma_{s}$ as follows:

$$
\int_{-\infty}^{+\infty} e^{i \xi x} \sigma_{s}(x) d x=\sum_{k=0}^{\infty} \frac{\left(-\xi^{2}\right)^{k}}{(2 k) !} m_{k}=\sum_{k=0}^{\infty} \beta_{k} \frac{\left(-\xi^{2}\right)^{k}}{k !},
$$

where

$$
\beta_{k}=\frac{1}{s k+1}\left(\begin{array}{c}
s k+k \\
k
\end{array}\right) \frac{k !}{(2 k) !}=\frac{1}{s k+1} \frac{(s k+k) !}{(s k) !(2 k) !} .
$$

A direct computation shows that the ratio

$$
\begin{aligned}
\frac{\beta_{k+1}}{\beta_{k}}= & \frac{s k+1}{s(k+1)+1} \frac{1}{(2 k+1)(2 k+2)} \\
& \times \frac{((s+1) k+1)((s+1) k+2) \cdots((s+1) k+s+1)}{(s k+1)(s k+2) \cdots(s k+s)} \\
= & \frac{K}{4} \frac{\left(k+\frac{1}{s+1}\right)\left(k+\frac{2}{s+1}\right) \cdots\left(k+\frac{s}{s+1}\right)}{\left(k+\frac{1}{2}\right)\left(k+\frac{2}{s}\right) \cdots\left(k+\frac{s}{s}\right)} \frac{1}{k+1+\frac{1}{s}} \\
& \doteq \frac{K}{4} \frac{\left(k+a_{1}\right)\left(k+a_{2}\right) \cdots\left(k+a_{s}\right)}{\left(k+b_{1}\right)\left(k+b_{2}\right) \cdots\left(k+b_{s}\right)} \frac{1}{k+b_{s+1}} .
\end{aligned}
$$


Here

$$
b_{1}=\frac{1}{2}, a_{i}=\frac{i}{s+1} \text { and } b_{i+1}=\frac{i+1}{s} \text { for } i=1,2, \ldots, s .
$$

Therefore, using the generalized hypergeometric function, we rewrite

$$
\begin{aligned}
\int_{-\infty}^{+\infty} e^{i \xi x} \sigma_{s}(x) d x & ={ }_{s} F_{s+1}\left(a_{1}, \ldots, a_{s} ; b_{1}, \ldots, b_{s}, b_{s+1} ;-\frac{K}{4} \xi^{2}\right) \\
& =\int_{[0,1]^{s}} F\left(t_{1}, \ldots, t_{s}\right)_{0} F_{1}\left(b_{s+1} ;-\frac{\tau K}{4} \xi^{2}\right) d^{s} t
\end{aligned}
$$

Note that $a_{1}=b_{1}$ for $s=1$ but $b_{j}>a_{j}>0, j=1,2, \ldots, s$, when $s>1$. In Eq. (0.13) we have made use of Euler's integral representation of the generalized hypergeometric function [2].

Next, with the help of the integral representation of the Bessel function of the first kind [2], that is, for $\alpha>-\frac{1}{2}$,

$$
\begin{aligned}
J_{\alpha}(z) & =\frac{(z / 2)^{\alpha}}{\Gamma(\alpha+1)}{ }_{0} F_{1}\left(\alpha+1 ;-\frac{1}{4} z^{2}\right) \\
& =\frac{(z / 2)^{\alpha}}{\sqrt{\pi} \Gamma\left(\alpha+\frac{1}{2}\right)} \int_{-1}^{1} e^{i z x}\left(1-x^{2}\right)^{\alpha-\frac{1}{2}} d x,
\end{aligned}
$$

we get

$$
\begin{aligned}
{ }_{0} F_{1}\left(\alpha+1 ;-\frac{1}{4} z^{2}\right) & =\frac{\Gamma(\alpha+1)}{\sqrt{\pi} \Gamma\left(\alpha+\frac{1}{2}\right)} \int_{-1}^{1} e^{i z x}\left(1-x^{2}\right)^{\alpha-\frac{1}{2}} d x \\
& =\frac{1}{B\left(\frac{1}{2}, \alpha+\frac{1}{2}\right)} \int_{-1}^{1} e^{i z x}\left(1-x^{2}\right)^{\alpha-\frac{1}{2}} d x .
\end{aligned}
$$

Set $\alpha=1 / s, z=\sqrt{\tau K} \xi$. We have

$$
\begin{aligned}
{ }_{0} F_{1}\left(b_{s+1} ;-\frac{\tau K}{4} \xi^{2}\right) & =\frac{1}{B\left(\frac{1}{2}, \frac{1}{2}+\frac{1}{s}\right)} \int_{-1}^{1} e^{i \sqrt{\tau K} \xi x}\left(1-x^{2}\right)^{\frac{1}{s}-\frac{1}{2}} d x \\
& =\frac{1}{B\left(\frac{1}{2}, \frac{1}{2}+\frac{1}{s}\right)} \int_{-\sqrt{\tau K}}^{\sqrt{\tau K}} e^{i \xi x} \frac{\left(\tau K-x^{2}\right)^{\frac{1}{s}-\frac{1}{2}}}{(\tau K)^{\frac{1}{s}}} d x .
\end{aligned}
$$

Combining (0.16) and (0.13) after interchanging the order of integration, we obtain

$$
\sigma_{s}(x)=\frac{1}{B\left(\frac{1}{2}, \frac{1}{2}+\frac{1}{s}\right)} \int_{[0,1]^{s}} \frac{\left(\tau K-x^{2}\right)^{1 / s-1 / 2}}{(\tau K)^{1 / s}} F\left(t_{1}, \ldots, t_{s}\right) 1_{\left\{\tau K \geq x^{2}\right\}} d^{s} t .
$$

Noting the fact that

$$
\pi_{s}(x)=\frac{\sigma_{s}(\sqrt{x})}{\sqrt{x}} 1_{(0, \infty)}
$$

the proof is then complete.

We remark that some properties of the density $\pi_{s}(x)$ follow easily from Theorem 0.1 for instance, there are no atoms, the support is $[0, K]$, and the density is analytic on $(0, K)$ (these properties were obtained earlier by implicit complexanalytic methods; see Theorem 2.1, 3]). 


\section{REFERENCES}

1. Alexeev, N., Götze, F., Tikhomirov, A.: Asymptotic distribution of singular values of powers of random matrices, Lithuanian Math. J. 50, no. 2 (2010), 121-132.

2. Andrews, G. E., Askey, R., Roy, R.: Special Functions, Cambridge University Press (1999). MR 1688958 (2000g:33001)

3. Banica, T., Belinschi, S., Capitaine, M., Collins, B.: Free Bessel laws, Canad. J. Math. 63 (2011), 3-37.

4. Collins, B., Nechita, I., Życzkowski, K.: Random graph states, maximal flow and Fuss-Catalan distributions, J. Phys. A: Math. Theor. 43 (2010), 275-303. MR2658283

5. Liu, D.-Z., Wang, Z.-D., Yan, K.-H.: Density of eigenvalues and its perturbation invariance in unitary ensembles of random matrices, Journal of Approximation Theory 162, no. 9 (2010), 1588-1606. MR2718887

6. Młtkowski, W.: Fuss-Catalan numbers in noncommutative probability, Documenta Mathematica 15 (2010), 939-955.

7. Nica, A., Speicher, R.: Lectures on the Combinatorics of Free Probability, Cambridge University Press (2006). MR2266879 (2008k:46198)

8. Penson, K. A., Solomon, A. I.: Coherent states from combinatorial sequences, arXiv:quant$\mathrm{ph} / 0111151 \mathrm{v} 1$

9. Wigner, E. P.: Characteristic vectors of bordered matrices with infinite dimensions, Ann. of Math. (2) 62 (1955), 548-564. MR0077805(17:1097c)

School of Mathematical Sciences, Lmam, Peking University, Beijing, 100871, PeoPLE'S Republic OF ChinA

Current address: Instituto Matemática y Física, Universidad de Talca, Casilla 747, Talca, Chile

E-mail address: dzliu@inst-mat.utalca.cl

School of Mathematical Sciences, LMam, Peking University, Beijing, 100871, PeoPLE'S REPUBLiC OF CHINA

E-mail address: csong@math.pku.edu.cn

School of Mathematical Sciences, LMaM, Peking University, Beijing, 100871, PeoPLE'S REPUBLIC OF CHINA

E-mail address: zdwang@pku.edu.cn 